Ingeborg W.M. van

Uden, MD

Helena M. van der Holst, MD

Esther M.C. van Leijsen, MSc

Anil M. Tuladhar, MD

Anouk G.W. van Norden, $\mathrm{MD}, \mathrm{PhD}$

Karlijn F. de Laat, MD, $\mathrm{PhD}$

Jurgen A.H.R. Claassen, $\mathrm{MD}, \mathrm{PhD}$

Ewoud J. van Dijk, MD, $\mathrm{PhD}$

Roy P.C. Kessels, PhD Edo Richard, MD, PhD Indira Tendolkar, MD, $\mathrm{PhD}$

Frank-Erik de Leeuw, $\mathrm{MD}, \mathrm{PhD}$

Correspondence to

Dr. de Leeuw:

frankerik.deleeuw@radboudumc.nl

Supplemental data at Neurology.org

\section{Late-onset depressive symptoms increase the risk of dementia in small vessel disease}

\section{ABSTRACT}

Objective: We prospectively investigated the role of depressive symptoms (DS) on all-cause dementia in a population with small vessel disease (SVD), considering onset age of DS and cognitive performance.

Methods: The RUN DMC study (Radboud University Nijmegen Diffusion Tensor and Magnetic Resonance Cohort) is a prospective cohort study among 503 older adults with SVD on MRI without dementia at baseline (2006), with a follow-up of 5 years (2012). Kaplan-Meier curves stratified for DS and dementia risk were compared using log-rank test. We calculated hazard ratios using Cox regression analyses.

Results: Follow-up was available for 496 participants (mean baseline age 65.6 years [SD 8.8]; mean follow-up time 5.2 years). All-cause dementia developed in 41 participants. The 5.5-year dementia risk was higher in those with DS (hazard ratio 2.7, 95\% confidence interval 1.45.2), independent of confounders. This was driven by those with late-onset DS. Five-year cumulative risk difference for dementia was higher in participants with depressive symptoms who had high baseline cognitive performance (no DS 0.0\% vs DS 6.9\%, log-rank $p<0.001$ ) compared with those who had low cognitive performance at baseline.

Conclusions: Late-onset DS increases dementia risk, independent of SVD. Especially in those with relatively high cognitive performance, DS indicate a higher risk. In contrast to current practice, clinicians should monitor those with DS who also show relatively good cognitive test scores.

Neurology ${ }^{\circledR}$ 2016;87:1102-1109

\section{GLOSSARY}

CES-D = Center for Epidemiologic Studies Depression Scale; CI = confidence interval; DS = depressive symptoms; DSMIV = Diagnostic and Statistical Manual of Mental Disorders (Fourth Edition); EODS = early-onset depressive symptoms; $\mathbf{G M}=$ gray matter; $\mathbf{H R}$ = hazard ratio; LODS = late-onset depressive symptoms; MMSE = Mini-Mental State Examination; MPRAGE = magnetization-prepared rapid-acquisition gradient echo; RUN DMC = Radboud University Nijmegen Diffusion Tensor and Magnetic Resonance Cohort; SAT = speed-accuracy trade-off; SVD = small vessel disease; TBV $=$ total brain volume; $\mathbf{W M}=$ white matter; $\mathbf{W M H}=$ white matter hyperintensity

Depressive symptoms (DS) occur in approximately $30 \%$ of patients with dementia ${ }^{1}$ and have been coined a risk factor for, ${ }^{2}$ an early symptom of, ${ }^{3}$ or a reaction to perceived cognitive decline. Both cross-sectional and longitudinal population-based studies have investigated this, showing conflicting results. ${ }^{4-8}$

Late-onset DS (LODS), initially occurring in older age, should be distinguished from earlyonset DS (EODS), for a different etiology is presumed. ${ }^{9,10}$ Particularly LODS, rather than EODS, were found to be related to incident dementia. ${ }^{11}$ It is unclear whether LODS increase dementia risk alone or whether this is mediated by the same underlying cerebral small vessel disease (SVD)., ${ }^{912-14}$ SVD refers to neuroradiologic findings, including white matter hyperintensities (WMH), lacunes of presumed vascular origin (lacunes), and microbleeds. ${ }^{15}$

One recent study in older adults with SVD has already shown that DS were associated with an increased risk of cognitive decline after 3 years, independent of WMH. ${ }^{16}$ However,

From the Donders Institute for Brain, Cognition and Behaviour, Radboud University Medical Centre, Departments of Neurology (I.W.M.v.U., H.M.v.d.H., E.M.C.v.L., A.M.T., E.J.v.D., E.R., F.-E.d.L.), Geriatrics (J.A.H.R.C.), Medical Psychology (R.P.C.K.), and Psychiatry (I.T.), Nijmegen; Department of Neurology (A.G.W.v.N.), Amphia Ziekenhuis Breda; and Department of Neurology (K.F.d.L.), HagaZiekenhuis The Hague, the Netherlands.

Go to Neurology.org for full disclosures. Funding information and disclosures deemed relevant by the authors, if any, are provided at the end of the article. 
important adjustments for other aspects of the SVD spectrum as well as gray matter (GM) and hippocampal volume were not made.

Therefore, we prospectively investigated the role of baseline DS on the risk of all-cause dementia in adults with SVD, also considering SVD characteristics and other well-known confounders. Second, we investigated the risk of dementia, stratified by age at onset of DS, as LODS and EODS might have different relations with incident dementia. Finally, we investigated the relation between DS and incident dementia in those with high and low cognitive performance at baseline.

METHODS Study population. The Radboud University Nijmegen Diffusion Tensor and Magnetic Resonance Cohort (RUN DMC study) is a prospective cohort study investigating risk factors and clinical consequences of brain changes during aging as assessed with MRI among 503 older adults without dementia aged 50 to 85 years with cerebral SVD. The recruitment, study rationale, and protocol were described previously in detail. ${ }^{17}$ SVD was defined on established research criteria (presence of any WMH and/or lacunes of presumed vascular origin), ${ }^{18}$ as SVD onset is often insidious and clinically heterogeneous with acute (TIAs or lacunar syndromes) or subacute (cognitive, motor, and/or mood disturbances) symptoms. Baseline data collection was performed in 2006. Main exclusion criteria were dementia, WMH, or SVD mimics, current (psychiatric) disease interfering with cognitive testing (such as current major depression) or follow-up, history of serious mental illness, and MRI contraindications (e.g., pacemaker, vascular clips, and known claustrophobia). ${ }^{17}$

Follow-up occurred in 2011-2012. Of 503 baseline participants, 2 were lost to follow-up (not deceased; figure e-1 at Neurology.org); 5 additional participants were excluded because of missing values on the Center for Epidemiologic Studies Depression Scale (CES-D), yielding a final sample of 496.

Standard protocol approvals, registrations, and patient consents. This study was approved by the Medical Review Ethics Committee region Arnhem-Nijmegen. All participants gave written informed consent before inclusion.

Assessment of DS. DS were assessed with the 20-item CES-D. ${ }^{19}$ DS were considered present when participants had a CES-D score $\geq 16$ and/or currently used antidepressants, taken for depression. Sixty-one participants used antidepressive medication..$^{20}$

Furthermore, participants were interviewed about their history of depressive episodes, onset age, and whether they asked for medical advice. If depressive episodes had required attention of a general practitioner, psychologist, or psychiatrist, a history of depression was considered present. ${ }^{21}$ To distinguish between LODS and EODS, we used the age of 60 as the cutoff point. ${ }^{21}$ Those with a CES-D score $\geq 16$ and/or current use of antidepressants, age 60 years or older, without a history of depressive episodes before the age of 60 , were classified as having LODS. Those with a CES-D score $<16$, without a history of DS and without current use of antidepressants, formed the reference group. The others (first depressive episode when younger than 60 years) fulfilled the criteria for EODS (table e-1). ${ }^{22}$

Dementia case finding. At baseline, all participants were free of dementia. Dementia case finding and assessment of time to dementia were described in detail previously. ${ }^{23,24}$ In short, dementia case finding was performed for all 501 participants: those who participated in the in-person follow-up and among those who could not (died or refused) (figure e-1). Participants who screened positive at in-person follow-up (MMSE score $\leq 26$ or a decline $\geq 3$ points from baseline) were subsequently examined for dementia at the Radboud Alzheimer Center. For those who refused this examination, the panel, consisting of members of the same Radboud Alzheimer Center (to ensure a standardized approach), came to a consensus diagnosis. This panel reviewed all available neuropsychological ${ }^{17,23}$ and imaging information next to interference in daily functioning, confirmed by family or caregivers.

For those who were not available for an in-person assessment, the same panel reviewed the medical records. Furthermore, we contacted patients' general practitioners and medical specialists for information on cognitive status. The dementia diagnosis was based on DSM-IV criteria. ${ }^{25}$ Incident dementia was diagnosed in 43 cases. Data on baseline DS were not available for 2 patients who developed dementia; therefore, 41 cases of incident dementia are included in the analysis (figure e-1).

MRI protocol and analyses. MRI scans were performed on a single 1.5-tesla MRI, on average 19 days after assessment at our research center. The protocol included, among other sequences, a T1-weighted 3-dimensional magnetization-prepared rapidacquisition gradient echo (MPRAGE) imaging (voxel size $1.0 \times$ $1.0 \times 1.0 \mathrm{~mm}$ ); fluid-attenuated inversion recovery (voxel size $1.0 \times 1.2 \times 5.0 \mathrm{~mm}$, with an interslice gap of $1 \mathrm{~mm}$ ); and a transversal $\mathrm{T} 2 *$-weighted gradient echo sequence (voxel size $1.3 \times 1.0 \times 6.0 \mathrm{~mm}$, with an interslice gap of $1 \mathrm{~mm}) .{ }^{17}$

WMH were manually segmented on fluid-attenuated inversion recovery images. Total WMH volume was calculated by summing the segmented areas multiplied by slice thickness. Lacunes and microbleeds were assessed by raters blinded to all clinical data, according to the Standards for Reporting Vascular Changes on Neuroimaging criteria, ${ }^{15}$ with good intra- and interrater variability. ${ }^{23}$ To obtain GM (cortex, basal ganglia, and thalamus), white matter (WM), and CSF volume, the T1 MPRAGE images were segmented using Statistical Parametric Mapping 12 unified segmentation routines (Wellcome Department of Cognitive Neurology, University College London, UK http://www.fil. ion.ucl.ac.uk/spm/software/spm12/). ${ }^{23}$ To obtain GM, WM, and CSF volumes, all voxel volumes that had a $p>0.5$ for belonging to that tissue class were summed. Intracranial volume was a summation of total GM, total WM, and CSF volume. Total brain volume (TBV) was a summation of GM and WM volume. We visually checked all images for coregistration errors and for motion and/or segmentation artifacts. Hippocampal volumes were manually segmented on the MPRAGE image using the interactive software program ITK-SNAP, as described previously ${ }^{26,27}$ (http://www.itksnap.org). To adjust for head size, all volumes were normalized to the intracranial volume.

Other measurements. Education was classified using 7 categories (1 represented less than primary school, 7 an academic degree). ${ }^{28}$ We then dichotomized in primary or less (levels 1-2) or more than primary education (levels 3-7). Speed-accuracy trade-off (SAT) scores were calculated where appropriate (accuracy [\%]/reaction time) to adjust for a number of faults. The Cognitive Index was constructed to obtain a more robust outcome measure for global cognition. In short, this was calculated as the mean of the $z$ scores of the SAT score of the Paper and Pencil Memory Scanning Task, the mean of the SAT score of the reading task of the Stroop test, the mean of the Symbol-Digit Substitution Task, and the mean of the added 
score on the 3 learning trials of the Rey Auditory Verbal Learning Test, and the mean of the Delayed Recall of this test. ${ }^{29}$ Functional independence was assessed using the Barthel Index (range, 020). ${ }^{17}$ All other measurements were done at initial assessment.

Statistical analyses. WMH volume was log-transformed because of the skewness of the data. Cumulative risk of incident dementia was estimated with Kaplan-Meier analysis stratified by presence of baseline DS. Person-years at risk were calculated for each participant from date of the baseline assessment, until onset of dementia, death, or date of the follow-up assessment. Those who did not reach the endpoint or died were censored. Subsequently, we used incidence curves to compare between subgroups using log-rank test. We used Cox regression analyses to calculate hazard ratios (HRs) with 95\% confidence intervals (CIs) for presence of baseline DS (total, EODS, and LODS), adjusted for age, sex, and education (model 1), additional for baseline Cognitive Index and TBV (model 2), additional for WMH volume, presence of lacunes and microbleeds (model 3), or additional for GM and hippocampal volume (model 4). Verification of proportionality of hazards was performed by examining Schoenfeld residuals; there were no indications that this assumption was violated. Finally, to identify the role of DS on the dementia risk in "high cognitive performers" and "low cognitive performers" at baseline (defined by compound $z$ score Cognitive Index below the median at baseline; table e-2), we performed Kaplan-Meier analyses and compared subgroups with log-rank test.

RESULTS Baseline demographic and neuroimaging characteristics $(n=496)$ are shown in tables 1 and e-3, respectively. At baseline, 33.7\% of participants had DS. Dementia developed in 41 of the 496 participants during a mean follow-up of 5.2 years (SD 0.7). Mean age at baseline was 65.6 years (SD 8.8), and $56.9 \%$ were men. Mean MMSE score at baseline was 28.1 (SD 1.6). The 5.5-year cumulative risk of dementia was higher in participants with DS at baseline compared with those without (DS 18.5\% vs no DS 7.4\%, log-rank $p=0.003$ ) (figure 1).

Baseline DS significantly predicted incident dementia after 5 years of follow-up, adjusted for age, sex, and education (HR 2.7, 95\% CI 1.4-5.2). Additional adjustment for baseline Cognitive Index and TBV, SVD characteristics (WMH volume, lacunes, and microbleeds), or GM structures (hippocampal and GM volume) did not significantly alter these findings (data not shown). The unadjusted cumulative risk of incident dementia in those with EODS and LODS separately compared to those who never experienced DS is presented in figure 2. LODS predicted incident dementia (HR 2.5, 95\% CI 1.34.8 ); adjustments for the above-mentioned confounders did not change this relation substantially (data not shown). For EODS, this relation with incident dementia was not found (table 2).

Finally, figure 3 shows the cumulative risk of dementia in strata of baseline cognitive performance for participants with and without DS. In depressed participants with high baseline cognitive performance, there was a significant 5 -year cumulative risk difference for dementia (no DS $0.0 \%$ vs DS 6.9\%,

Table 1 Baseline characteristics of participants with and without depressive symptoms at baseline assessment

\begin{tabular}{|c|c|c|c|c|}
\hline Characteristic & Total $(\mathrm{N}=496)$ & $\begin{array}{l}\text { Depressive symptoms at } \\
\text { baseline }(n=167)\end{array}$ & $\begin{array}{l}\text { No depressive symptoms at } \\
\text { baseline }(n=329)\end{array}$ & $\begin{array}{l}\text { p Value for } \\
\text { difference }^{a}\end{array}$ \\
\hline Age at baseline (SD), y & $65.6(8.8)$ & $65.9(8.7)$ & $65.4(8.8)$ & $0.607^{b}$ \\
\hline Men, n (\%) & $282(56.9)$ & $81(48.5)$ & $201(61.1)$ & $0.007^{c}$ \\
\hline Only primary education, n (\%) & $48(9.7)$ & $26(15.6)$ & $22(6.7)$ & $0.002^{c}$ \\
\hline CES-D score at baseline (SD) & $11.0(9.4)$ & $21.1(8.5)$ & $5.9(4.4)$ & $0.001^{d}$ \\
\hline Antidepressive medication use at baseline, $n(\%)$ & 61 (12.3) & 61 (36.5) & $0(0.0)$ & NA \\
\hline History of depression, $\mathrm{n}(\%)$ & $127(25.6)$ & 85 (50.9) & $42(12.8)$ & $0.001^{c}$ \\
\hline MMSE baseline score (SD) & $28.1(1.6)$ & $27.7(1.7)$ & $28.3(1.6)$ & $<0.001^{d}$ \\
\hline Compound z score Cognitive Index (SD) ${ }^{e}$ & $-0.0(0.8)$ & $-0.3(0.7)$ & $0.1(0.8)$ & $<0.001^{\mathrm{b}}$ \\
\hline Low cognitive performance, $n(\%)^{e}$ & $247(49.8)$ & $107(64.1)$ & $140(42.6)$ & $<0.001^{\mathrm{c}}$ \\
\hline Dementia at follow-up, $n$ (\%) & 41 (8.3) & 22 (13.2) & $19(5.8)$ & $0.005^{c}$ \\
\hline Barthel Index (SD) & $19.7(0.8)$ & $19.5(1.1)$ & $19.8(0.6)$ & $0.011^{b}$ \\
\hline
\end{tabular}

Abbreviations: CES-D = Center for Epidemiologic Studies Depression Scale; MMSE = Mini-Mental State Examination.

Data shown are unadjusted values and represent $n$ (\%) and mean (SD). Of the 503 participants at baseline, 2 were lost to follow-up. Five additional participants were excluded because of missing values on the status of depression at baseline (one was excluded because of missing cognitive tests at baseline). The Cognitive Index is a compound $z$ score of the paper and pencil test, Stroop test, Symbol-Digit Substitution Task, and immediate and delayed recall of the 15-word list of the Rey Auditory Verbal Learning Test. Low cognitive performance is defined by a compound $z$ score of the Cognitive Index below the median at baseline.

${ }^{a}$ Comparison of those with and without depressive symptoms at baseline.

${ }^{\mathrm{b}}$ Means compared with independent $t$ test.

${ }^{\mathrm{c}}$ Chi-square test.

${ }^{\mathrm{d}}$ Mann-Whitney U.

e One participant was excluded because of missing cognitive tests at baseline. 
Figure 1 Cumulative risk of dementia stratified by presence of depressive symptoms at baseline

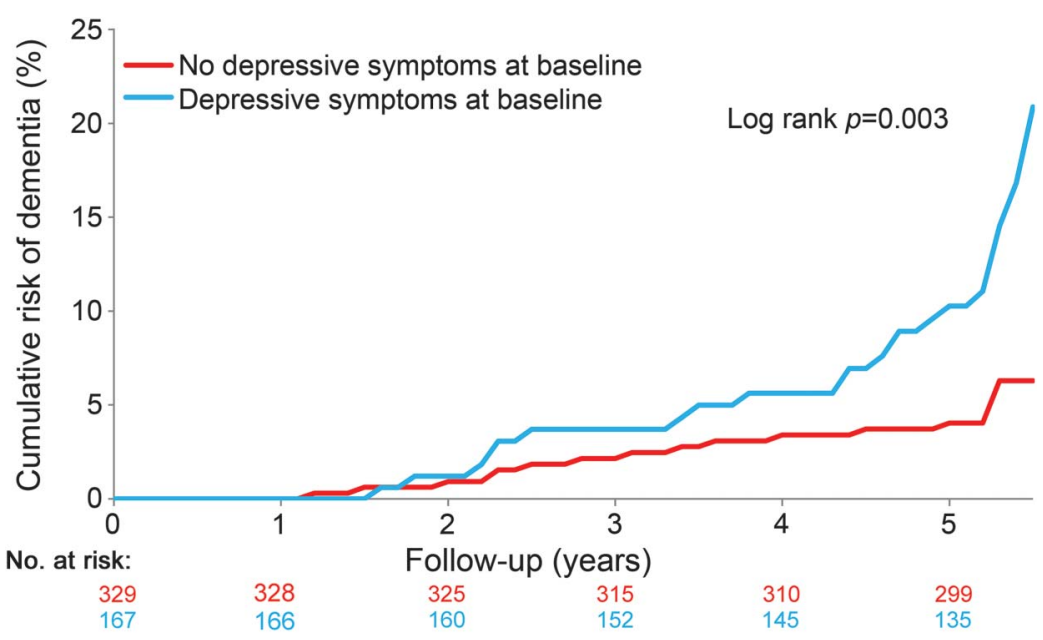

Unadjusted percentages. Log-rank test pairwise over strata.

$\log$-rank $p<0.001)$, whereas no such difference was found between participants with and without DS with low cognitive performance (no DS $9.7 \%$ vs DS $12.2 \%, \log$-rank $p=0.514$ ). Adjustment for demographic and imaging confounders in Cox regression analyses in both good and poor cognitive performance did not change this relation. Low dementia risk in participants without DS with good cognitive performance led to wide CIs (HR 40.2, 95\% CI 4.5361.8).

The interaction between DS and cognitive performance on dementia risk showed a significant effect (HR 25.3, 95\% CI 2.6-238.7; $p=0.005$ ). We did not find a significant interaction between sex or low education and DS (data not shown).

DISCUSSION Our results show that DS increase the dementia risk after 5 years in older adults with SVD, a relation that is driven by those who develop a first depressive episode at later age. This relation is independent of SVD characteristics and GM and hippocampal volumes, suggesting additional factors besides SVD have a role in our cohort in development of dementia. We showed that DS are related to an increased risk of dementia in those without objective cognitive problems. The magnitude of this relation, however, cannot be estimated because of the small number of incident cases in this subgroup. Below, we discuss the significance of these findings in more detail.

Strengths of our study include its longitudinal and single-center design allowing us to collect both baseline and follow-up data according to identical procedures, reducing the risk of procedural bias. Subsequently, the large cohort that covers the whole spectrum of cerebral SVD and the high follow-up rate

Figure 2 Cumulative risk of dementia stratified by onset of depressive symptoms

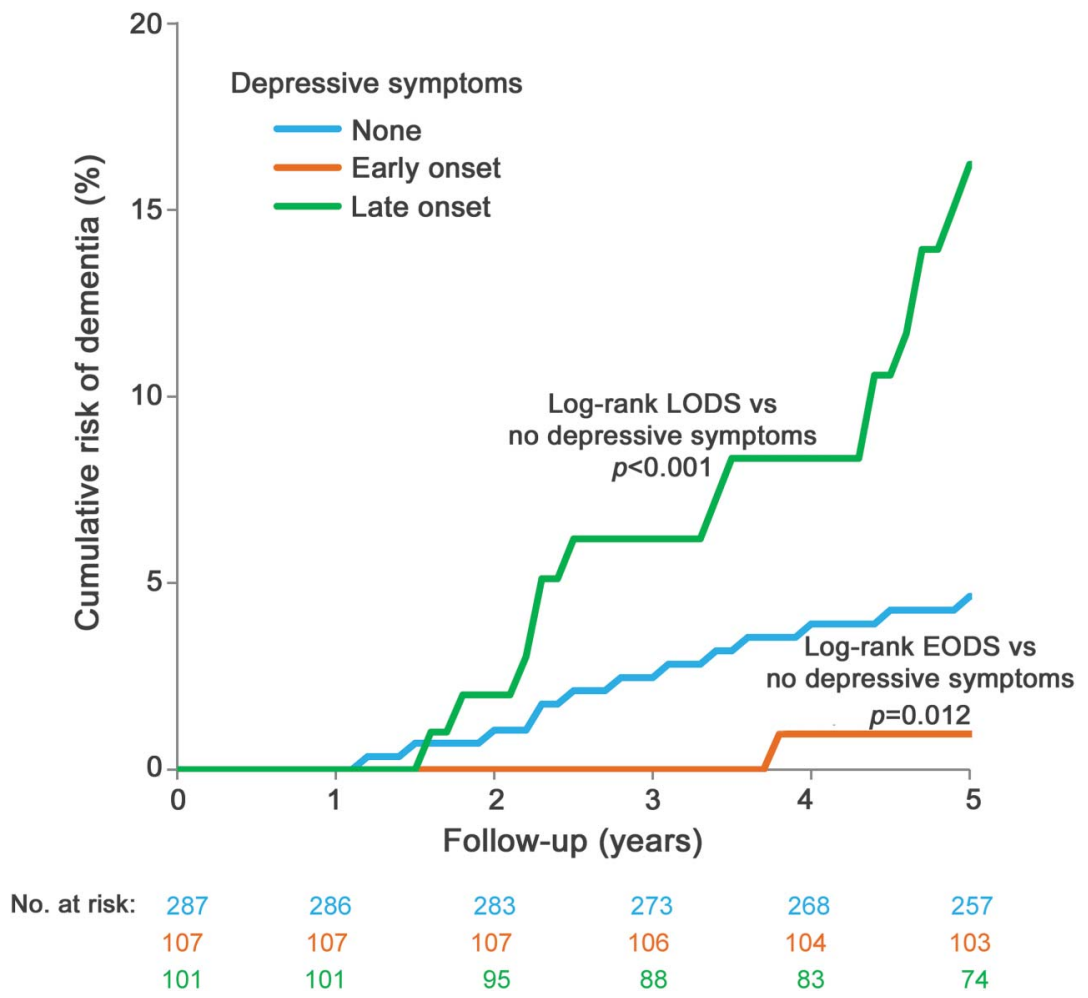

Unadjusted percentages. Log-rank test pairwise over strata. EODS = early-onset depressive symptoms; LODS = late-onset depressive symptoms. 


\begin{tabular}{|c|c|c|c|}
\hline \multirow[t]{2}{*}{ Table 2} & \multicolumn{3}{|c|}{$\begin{array}{l}\text { Cox proportional hazards for dementia by baseline depression status, } \\
\text { early-onset depressive symptoms, and late-onset depressive } \\
\text { symptoms }\end{array}$} \\
\hline & & $\begin{array}{l}\text { Progression to } \\
\text { dementia, n (\%) }\end{array}$ & $\begin{array}{l}\text { HR }(95 \% \mathrm{Cl}) \text {, } \\
\text { adjusted for age, } \\
\text { sex, and education }\end{array}$ \\
\hline \multicolumn{4}{|c|}{ Depressive symptoms } \\
\hline \multicolumn{2}{|c|}{ No depressive symptoms $(n=329)$} & $19(5.8)$ & 1 \\
\hline \multicolumn{2}{|c|}{ Depressive symptoms $(n=167)$} & $22(13.2)$ & $2.7(1.4-5.2)^{\mathrm{a}}$ \\
\hline \multicolumn{4}{|c|}{ Early-onset $(<60$ y) } \\
\hline \multicolumn{2}{|c|}{ No depressive symptoms $(n=287)$} & $19(6.6)$ & 1 \\
\hline \multicolumn{2}{|c|}{ Early-onset depressive symptoms $(n=107)$} & $1(0.9)$ & $0.2(0.02-1.7)$ \\
\hline \multicolumn{4}{|c|}{ Late-onset ( $\geq 60$ y) } \\
\hline \multicolumn{2}{|c|}{ No depressive symptoms $(n=287$ ) } & $19(6.6)$ & 1 \\
\hline \multicolumn{2}{|c|}{ Late-onset depressive symptoms ( $\mathrm{n}=101$ ) } & 21 (20.6) & $2.5(1.3-4.8)^{\mathrm{a}}$ \\
\hline
\end{tabular}

Abbreviations: $\mathrm{Cl}=$ confidence interval; $\mathrm{HR}=$ hazard ratio.

Of the 503 participants at baseline, 2 were lost to follow-up. Five additional participants were excluded because of missing values on the status of depression at baseline resulting in a total of 496 participants. Late-onset depressive symptoms: patients aged 60 years or older with a Center for Epidemiologic Studies Depression Scale (CES-D) score $\geq 16$ and/or current use of antidepressive medication, taken for depression, without a history of depressive episodes when younger than 60 years. Early-onset depressive symptoms: a CES-D score $\geq 16$ and/or current use of antidepressive medication, taken for depression, and/or a history of depressive episodes when younger than 60 years. Individuals with a CES-D score $<16$, without a history of depressive symptoms and without current use of antidepressive medication, formed the reference group. Verification of proportionality of hazards was performed by examining Schoenfeld residuals. a Significant.

of $99.6 \%$ are major strengths. We manually segmented WMH and hippocampal volumes without prior knowledge of clinical data. Finally, the relation between DS and incident dementia was investigated with adjustment for demographics, baseline global cognition, SVD, and GM structures, reducing confounds.

Several methodological issues need to be addressed. First, we investigated "overall dementia" as an outcome measure and did not investigate the risk of Alzheimer disease and vascular dementia separately. This distinction is difficult to make in elderly persons since at a later age vascular diseases and neurodegeneration often co-occur. ${ }^{30}$ Our dementia diagnosis was a clinical diagnosis, supported by MRI most closely performed to the moment of diagnosis. It is possible that we have missed a few cases of incident dementia in participants who were not available for in-person follow-up, because of incomplete medical records or uninformed general practitioners. In those cases, misclassification could have occurred in relatively early stages of dementia. Second, although widely used, the cutoff point of 26 in the MMSE might not be sensitive enough to detect all incident dementia cases, especially for those in an early stage of the disease, those with vascular dementia, or in participants with dementia who have higher education levels. Therefore, misclassification, which might have led to an underestimation of the effect, might have occurred. Third, the relation between DS and incident all-cause dementia was adjusted for demographic and imaging characteristics and for baseline cognitive performance, reducing confounders. However, the small number of dementia cases in some of the subgroups resulted in wide confidence intervals, preventing a reliable estimate of the magnitude of the effect. Therefore, these results should be interpreted with

Figure 3 Cumulative risk of dementia stratified by presence of depressive symptoms in participants with high and low cognitive performance at baseline
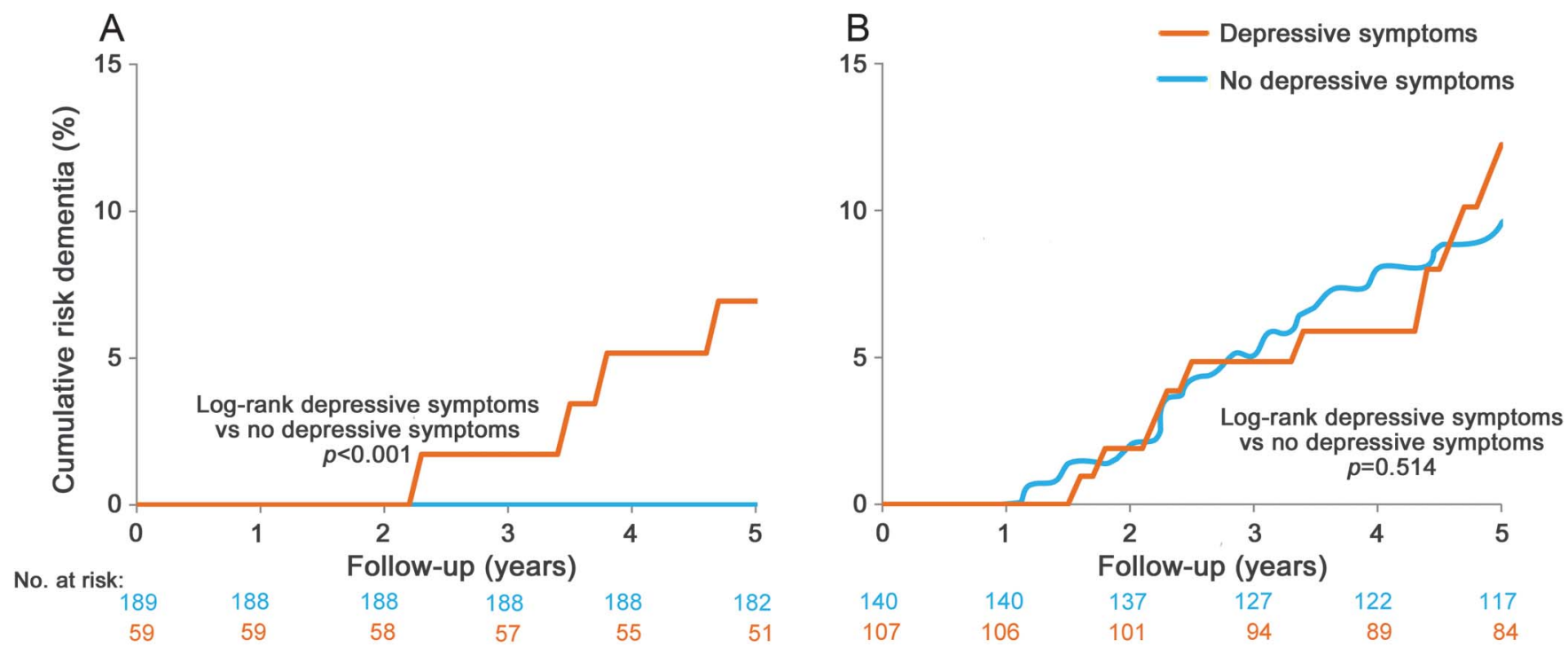

Cumulative risk of dementia in participants with high (A) and low (B) cognitive performance at baseline. Unadjusted percentages. Log-rank test pairwise over strata. High cognitive performance defined as the upper-half Cognitive Index at baseline; low cognitive performance defined as the lower-half Cognitive Index at baseline. 
caution. Furthermore, our analyses were not adjusted for other relevant confounders such as lifestyle risk factors and functional health, which might have led to residual confounding. Finally, we used the CES-D and/or current use of antidepressive medication to assess DS. Although used in large population-based studies, ${ }^{4,31}$ it is also considered a screen for general affective distress, and therefore not a specific screening tool for DS. To account for this, we excluded participants at baseline with serious mental illness.

We think that our study has an excellent external validity regarding vulnerability for cognitive and affective symptoms in patients between 50 and 85 years of age presenting with SVD on neuroimaging in a neuropsychiatric clinic. All of our participants were functionally independent at baseline, with a mean MMSE score of 28.1 (SD 1.6), reflecting estimates in the general population. The incidence rate for dementia in our SVD cohort is higher than in a large population-based study. ${ }^{32}$ This is not surprising as SVD is associated with incident dementia. ${ }^{12}$ When we compared our incidence rate of dementia with another SVD cohort, ${ }^{16}$ our incidence rate was actually lower. This could be explained by the approximately 10 years younger mean age of our population and also the less severe WMH at baseline; both factors are known to be related to the incidence of dementia. ${ }^{32,33}$

Our findings that DS are associated with all-cause dementia are accounted for by those participants with LODS. At the same time, we showed that EODS were not related to incident dementia in older adults with SVD. This is an important finding because (longer existing) EODS would be expected to increase dementia risk, for they have shown to be associated with neurodegeneration through neurotoxic mechanisms, including hippocampus atrophy. ${ }^{34,35}$ This could suggest that LODS present not a causal factor for incident dementia, but rather are an early symptom of the spectrum, ultimately leading to dementia, for they present relatively shortly before dementia onset.

There are several hypothesized explanations for (late-onset) DS as an early symptom of dementia. ${ }^{36}$ Disruption of the subcortical-frontal circuit, for example, by SVD or neurodegenerative processes could result in both LODS and cognitive difficulties, such as dysexecutive problems and slower speed of information processing. ${ }^{20,37-39}$ This structural defect might also explain resistance to treatment, which is common in late-life depression. Of note, in our study we found that the association between DS and incident dementia remained present after adjustment for SVD characteristics, suggesting that additional factors besides SVD have a role that may not be necessarily depicted at the macroanatomical level. This is in line with other longitudinal studies on depression and dementia. ${ }^{16,40}$

We showed in an SVD population that participants with DS have a higher risk of incident dementia compared to those without DS while performing relatively well cognitively. This finding could not be merely explained by the level of education, as post hoc analyses showed that all of those with incident dementia in the group that performed relatively well cognitively had more than primary education (data not shown). While it seems somewhat counterintuitive at first glance, it may very well be that only in those with relatively high cognitive performance, DS are indicative of incident dementia. Because cognitive performance at baseline is a very strong risk factor for incident dementia, it is possible that in the group with relatively low cognitive performance, presence of DS adds no significant value to the overall dementia risk. This is another argument suggesting that both (cognitive and affective) symptoms are part of the same underlying pathophysiology, whereby DS may present an early symptom rather than a risk factor for dementia.

The information presented above could imply that the presence of DS might be a sensitive tool to investigate future dementia risk, possibly more sensitive than cognitive evaluation, in those with a relatively higher cognitive status. In contrast to current clinical practice, these patients in particular should be adequately monitored for occurrence of cognitive decline, while those without DS have a very low 5-year risk of developing dementia. This is important to acknowledge, beginning with the general practitioner, who must recognize these first DS at older age as an initial sign of dementia. After this, the diagnostic path calls for a multidisciplinary approach in which there must be good cooperation among psychiatrists, clinical neuropsychologists, and neurologists.

Our results show that LODS increase the risk of all-cause dementia in elderly persons with SVD, but independent thereof, suggesting that other factors also have a role. It is possible that DS in older age are an early symptom rather than an independent risk factor for incident dementia. General practitioners and medical specialists should be alert to the presence of DS and also monitor those with relatively good cognitive test scores while presenting with DS.

\section{AUTHOR CONTRIBUTIONS}

Dr. van Uden: involved in data collection, analysis and interpretation of data, and drafting and revising the manuscript. Dr. van der Holst: involved in data collection and in revising the manuscript. Dr. van Leijsen: involved in data collection and in revising the manuscript. Dr. Tuladhar: involved in data collection and in revising the manuscript. Dr. van Norden: involved in study concept and design, data collection, and revising the manuscript. Dr. de Laat: involved in study concept and design, data collection, and revising the manuscript. Dr. Claassen: involved in study concept and design and revising the manuscript. 
Dr. van Dijk: involved in study concept and design and revising the manuscript. Dr. Kessels: involved in study concept and design and revising the manuscript. Dr. Richard: involved in analysis and interpretation of data and revising the manuscript. Dr. Tendolkar: involved in analysis and interpretation of data and revising the manuscript. Dr. de Leeuw: involved in study concept and design, analysis and interpretation of data, revising the manuscript, study supervision, and obtaining funding.

\section{STUDY FUNDING}

This work was supported by a VIDI innovational grant from the Netherlands Organisation for Scientific Research (NWO, grant 016.126.351) awarded to F.E.d.L. and the MIRA Institute for Biomedical Technology and Technical Medicine, University of Twente.

\section{DISCLOSURE}

I. van Uden, H. van der Holst, E. van Leijsen, A. Tuladhar, A. van Norden, K. de Laat, and J. Claassen report no disclosures relevant to the manuscript. E. van Dijk received a personal fellowship from the Dutch Brain Foundation (H04-12; F2009[1]-16). R. Kessels, E. Richard, and I. Tendolkar report no disclosures relevant to the manuscript. F. de Leeuw was awarded a VIDI innovational grant from the Netherlands Organisation for Scientific Research (NWO, grant 016.126.351); the study was also supported by the MIRA Institute for Biomedical Technology and Technical Medicine, University of Twente. Go to Neurology.org for full disclosures.

Received December 10, 2015. Accepted in final form May 30, 2016

\section{REFERENCES}

1. Chi S, Wang C, Jiang T, Zhu XC, Yu JT, Tan L. The prevalence of depression in Alzheimer's disease: a systematic review and meta-analysis. Curr Alzheimer Res 2015; 12:189-198.

2. Green RC, Cupples LA, Kurz A, et al. Depression as a risk factor for Alzheimer disease: the MIRAGE Study. Arch Neurol 2003;60:753-759.

3. Masters MC, Morris JC, Roe CM. Noncognitive symptoms of early Alzheimer disease: a longitudinal analysis. Neurology 2015;84:617-622.

4. Becker JT, Chang YF, Lopez OL, et al. Depressed mood is not a risk factor for incident dementia in a communitybased cohort. Am J Geriatr Psychiatry 2009;17:653-663.

5. Ownby RL, Crocco E, Acevedo A, John V, Loewenstein D. Depression and risk for Alzheimer disease: systematic review, meta-analysis, and metaregression analysis. Arch Gen Psychiatry 2006;63:530-538.

6. Byers AL, Yaffe K. Depression and risk of developing dementia. Nat Rev Neurol 2011;7:323-331.

7. Diniz BS, Butters MA, Albert SM, Dew MA, Reynolds CF III. Late-life depression and risk of vascular dementia and Alzheimer's disease: systematic review and meta-analysis of community-based cohort studies. $\mathrm{Br} \mathrm{J}$ Psychiatry 2013;202:329-335.

8. Richard E, Reitz C, Honig LH, et al. Late-life depression, mild cognitive impairment, and dementia. JAMA Neurol 2013;70:374-382.

9. Mendlewicz J, Baron M. Morbidity risks in subtypes of unipolar depressive illness: differences between early and late onset forms. Br J Psychiatry 1981;139:463-466.

10. Krishnan KR, McDonald WM, Doraiswamy PM, et al. Neuroanatomical substrates of depression in the elderly. Eur Arch Psychiatry Clin Neurosci 1993;243:41-46.

11. da Silva J, Goncalves-Pereira M, Xavier M, MukaetovaLadinska EB. Affective disorders and risk of developing dementia: systematic review. Br J Psychiatry 2013;202:177-186.

12. Prins ND, Scheltens P. White matter hyperintensities, cognitive impairment and dementia: an update. Nat Rev Neurol 2015;11:157-165.
13. Herrmann LL, Le Masurier M, Ebmeier KP. White matter hyperintensities in late life depression: a systematic review. J Neurol Neurosurg Psychiatry 2008;79:619-624.

14. van Sloten TT, Sigurdsson S, van Buchem MA, et al. Cerebral small vessel disease and association with higher incidence of depressive symptoms in a general elderly population: the AGES-Reykjavik Study. Am J Psychiatry 2015;172:570-578.

15. Wardlaw JM, Smith EE, Biessels GJ, et al. Neuroimaging standards for research into small vessel disease and its contribution to ageing and neurodegeneration. Lancet Neurol 2013;12:822-838.

16. Verdelho A, Madureira S, Moleiro C, et al. Depressive symptoms predict cognitive decline and dementia in older people independently of cerebral white matter changes: the LADIS Study. J Neurol Neurosurg Psychiatry 2013;84: 1250-1254.

17. van Norden AG, de Laat KF, Gons RA, et al. Causes and consequences of cerebral small vessel disease. The RUN DMC Study: a prospective cohort study. Study rationale and protocol. BMC Neurol 2011;11:29.

18. Erkinjuntti T. Subcortical vascular dementia. Cerebrovasc Dis 2002;13(suppl 2):58-60.

19. Radloff LS. The CES-D scale: a self report depression scale for research in the general population. Appl Psychol Meas 1977;1:385-401.

20. van Uden IW, Tuladhar AM, de Laat KF, et al. White matter integrity and depressive symptoms in cerebral small vessel disease: the RUN DMC Study. Am J Geriatr Psychiatry 2014;23:525-535.

21. de Groot JC, de Leeuw FE, Oudkerk M, Hofman A, Jolles J, Breteler MM. Cerebral white matter lesions and depressive symptoms in elderly adults. Arch Gen Psychiatry 2000;57:1071-1076.

22. van Uden IW, van Norden AG, de Laat KF, et al. Depressive symptoms and amygdala volume in elderly with cerebral small vessel disease: the RUN DMC Study. J Aging Res 2011;2011:647869.

23. van Uden IW, Tuladhar AM, van der Holst HM, et al. Diffusion tensor imaging of the hippocampus predicts the risk of dementia; the RUN DMC Study. Hum Brain Mapp 2016;37:327-337.

24. van Uden IW, van der Holst HM, Tuladhar AM, et al. White matter and hippocampal volume predict the risk of dementia in patients with cerebral small vessel disease: the RUN DMC Study. J Alzheimers Dis 2015;49:863-873.

25. American Psychiatric Association. Diagnostic and Statistical Manual of Mental Disorders: DSM-IV. Washington, DC: American Psychiatric Association; 2000.

26. van Norden AG, Fick WF, de Laat KF, et al. Subjective cognitive failures and hippocampal volume in elderly with white matter lesions. Neurology 2008;71:1152-1159.

27. Yushkevich PA, Piven J, Hazlett HC, et al. User-guided 3D active contour segmentation of anatomical structures: significantly improved efficiency and reliability. Neuroimage 2006;31:1116-1128.

28. Hochstenbach J, Mulder T, van Limbeek J, Donders R, Schoonderwaldt H. Cognitive decline following stroke: a comprehensive study of cognitive decline following stroke. J Clin Exp Neuropsychol 1998;20:503-517.

29. van Uden IW, van der Holst HM, Schaapsmeerders P, et al. Baseline white matter microstructural integrity is not related to cognitive decline after 5 years: the RUN DMC Study. BBA Clin 2015;4:108-114. 
30. Viswanathan A, Rocca WA, Tzourio C. Vascular risk factors and dementia: how to move forward? Neurology 2009;72:368-374.

31. Saczynski JS, Beiser A, Seshadri S, Auerbach S, Wolf PA, Au R. Depressive symptoms and risk of dementia: the Framingham Heart Study. Neurology 2010;75:35-41.

32. Ott A, Breteler MM, van Harskamp F, Stijnen T, Hofman A. Incidence and risk of dementia: the Rotterdam Study. Am J Epidemiol 1998;147:574-580.

33. Prins ND, van Dijk EJ, den Heijer T, et al. Cerebral white matter lesions and the risk of dementia. Arch Neurol 2004;61:1531-1534.

34. Mulsant BH, Pollock BG, Kirshner M, Shen C, Dodge H, Ganguli M. Serum anticholinergic activity in a communitybased sample of older adults: relationship with cognitive performance. Arch Gen Psychiatry 2003;60:198-203.

35. Jacobson L, Sapolsky R. The role of the hippocampus in feedback regulation of the hypothalamic-pituitaryadrenocortical axis. Endocr Rev 1991;12:118-134.
36. Butters MA, Young JB, Lopez O, et al. Pathways linking late-life depression to persistent cognitive impairment and dementia. Dialogues Clin Neurosci 2008;10:345-357.

37. Lesser IM, Boone KB, Mehringer CM, Wohl MA, Miller BL, Berman NG. Cognition and white matter hyperintensities in older depressed patients. Am J Psychiatry 1996;153:1280-1287.

38. Alexopoulos GS. Frontostriatal and limbic dysfunction in late-life depression. Am J Geriatr Psychiatry 2002;10: 687-695.

39. Gudmundsson P, Olesen PJ, Simoni M, et al. White matter lesions and temporal lobe atrophy related to incidence of both dementia and major depression in 70-year-olds followed over 10 years. Eur J Neurol 2015;22:781-788, e49-e 50

40. Luchsinger JA, Honig LS, Tang MX, Devanand DP. Depressive symptoms, vascular risk factors, and Alzheimer's disease. Int J Geriatr Psychiatry 2008;23: 922-928.

\section{Complimentary CME and Self-assessment Resources: An Exclusive AAN Membership Benefit!}

Looking for ways to earn important CME credits in 2016? Look no further, because AAN membership provides FREE* access to the AAN's suite of online learning programs: NeuroSAE ${ }^{\circledR}$, NeuroLearn $^{\mathrm{SM}}$, and NeuroPI ${ }^{\mathrm{SM}}$. Access from virtually anywhere-home or office-to meet your CME needs, as well as take the necessary steps toward fulfilling your maintenance of certification (MOC) requirements, as mandated by the ABPN. Visit $A A N$.com/view/MOC today!

*Free access is limited to one course per program at a time.

\section{Call for Nominations: Editor-in-Chief of Neurology Today}

The AAN seeks self-nominations or nominations of other AAN members for the editor-in-chief of Neurology Today ${ }^{\circledR}$. The Academy's official news source publishes twice a month reporting on breaking news, issues, and trends in the practice and neurology, reaching over 26,000 professionals.

The editor-in-chief serves as the leader setting the future editorial vision and direction for the publication while continuing the strong tradition of providing reliable, accurate, neurologist edited and curated news covering the field of neurology.

The initial appointment is five years beginning July 1, 2017, with a two-month transition with the current editor-in-chief beginning April 1, 2017. The deadline for nominations is October 31, 2016. A position description, including requirements, is available at AAN.com/view/NTEditorInChief. 


\section{Neurology}

Late-onset depressive symptoms increase the risk of dementia in small vessel disease Ingeborg W.M. van Uden, Helena M. van der Holst, Esther M.C. van Leijsen, et al. Neurology 2016;87;1102-1109 Published Online before print August 12, 2016 DOI 10.1212/WNL.0000000000003089

This information is current as of August 12, 2016

\author{
Updated Information \& \\ Services
}

Supplementary Material

\section{References}

Subspecialty Collections

Permissions \& Licensing

Reprints including high resolution figures, can be found at: http://n.neurology.org/content/87/11/1102.full

Supplementary material can be found at: http://n.neurology.org/content/suppl/2016/08/14/WNL.0000000000003 089.DC1

This article cites 39 articles, 9 of which you can access for free at: http://n.neurology.org/content/87/11/1102.full\#ref-list-1

This article, along with others on similar topics, appears in the following collection(s):

All Cognitive Disorders/Dementia

http://n.neurology.org/cgi/collection/all_cognitive_disorders_dementia Cohort studies

http://n.neurology.org/cgi/collection/cohort_studies

Depression

http://n.neurology.org/cgi/collection/depression

MRI

http://n.neurology.org/cgi/collection/mri

Other cerebrovascular disease/ Stroke

http://n.neurology.org/cgi/collection/other_cerebrovascular_disease_st roke

Information about reproducing this article in parts (figures,tables) or in its entirety can be found online at:

http://www.neurology.org/about/about_the_journal\#permissions

Information about ordering reprints can be found online:

http://n.neurology.org/subscribers/advertise

Neurology ${ }^{\circledR}$ is the official journal of the American Academy of Neurology. Published continuously since 1951 , it is now a weekly with 48 issues per year. Copyright @ 2016 American Academy of Neurology. All rights reserved. Print ISSN: 0028-3878. Online ISSN: 1526-632X.

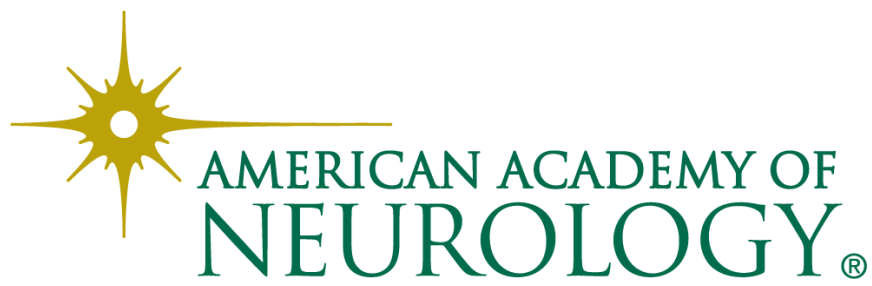

